\title{
Neuropsychiatric Manifestations as the Presentation of Addison's Disease Masquerading the Illness
}

\author{
Authors \\ Abiramy.S.P. ${ }^{1}$, Vengadakrishnan.K ${ }^{2}$ \\ ${ }^{1}$ Resident, Dept. of General Medicine, Sri Ramachandra Medical College, Porur, Chennai \\ ${ }^{2}$ Professor, Dept. of General Medicine, Sri Ramachandra Medical College, Porur, Chennai \\ Corresponding Author
}

Dr K. Vengadakrishnan

Sri Ramachandra Medical College, Porur, Chennai

Email:drkvk1975@gmail.com Phone numbers: 9840131997

\begin{abstract}
Adrenal insufficiency may present acutely as adrenal crisis or with chronic nonspecific symptoms, which requires a great deal of clinical suspicion. Adrenal insufficiency may also manifest with psychiatric manifestations, which makes its diagnosis even more challenging. The neuropsychiatric aspect of this condition is not fully understood. Patients with psychiatric symptoms are usually screened for other organic causes and are labeled as having mental disturbance. Neuropsychiatric symptoms could be the first presentation of Addison's disease. We present a 38 year old female who initially presented with vomiting and altered behavior, subsequently diagnosed to have hypopituitarism.
\end{abstract}

Keywords: Addison's disease, Hypopituitarism, Neuropsychiatric manifestations,

\section{Introduction}

Adrenal insufficiency is a disorder caused due to the deficiency of predominantly glucocorticoid and sometimes mineralocorticoid deficiency. It may present acutely as adrenal crisis or with chronic nonspecific symptoms, which requires a great deal of clinical suspicion. Adrenal insufficiency may also manifest with psychiatric manifestations, which makes its diagnosis even more challenging. The neuropsychiatric aspect of this condition is not fully understood and literatures have emphasized the need for endocrine workup. Patients with psychiatric symptoms are usually screened for other organic causes and are labeled as having mental disturbance, without identifying underlying adrenal insufficiency. Neuropsychiatric symptoms could be the first presentation of Addison's disease, and thus should be kept in mind whenever such a case presents to the physician. We present this case in which the patient initially demonstrated neuropsychiatric symptoms leading to a misdiagnosis of psychiatric illness, who showed dramatic response to therapy, after a definitive diagnosis was made.

\section{Case report}

A 38 year old female presented to our hospital with complaints of 2 episodes of vomiting, 4 
episodes of watery, non bloody stools and irritable behavior for 3 days. Patient did not have any history of fever, abdominal pain, jaundice or seizures during the time of presentation to the hospital. Patients had similar episodes of altered behavior for the past six months, for which she was on anti-psychotic medications. Patient did not have any other comorbid illness or history of surgery in the past. Patient had two normal vaginal deliveries, the second of which was associated with postpartum hemorrhage requiring multiple blood transfusions. Patient had lactation failure during her second puerperal period. Patient had normal menstrual cycles since and no other gynecological issues. She was on anti-psychotic medications for the past 6 months following complaints by her husband that she was irritable at home with impaired sleep and having delusional thoughts.

On examination, patient was found to be drowsy with mild dehydration, with a blood pressure of $70 / 40 \mathrm{~mm} \mathrm{Hg}$, pulse rate of 94/min. Systemic examination did not reveal any abnormality. Patient was resuscitated in the intensive care unit with a total of 2 litre bolus of normal saline. Patient continued to be hypotensive in spite of fluid resuscitation and hence started on noradrenaline support. Laboratory investigations revealed a hemoglobin of $10.1 \mathrm{gm} \%$, ESR of $33 \mathrm{~mm} / \mathrm{hr}$, RBS of $58 \mathrm{mg} \%$, sodium of $121 \mathrm{mmol} / \mathrm{l}$ and chloride of $87 \mathrm{mmol} / \mathrm{l}$. In view of hyponatremia, hypoglycemia and distributive shock not responding to fluid resuscitation, a diagnosis of adrenal insufficiency was considered. Random serum cortisol done was $2.60 \mathrm{mcg} / \mathrm{dl}$. Hence, patient was diagnosed as having adrenal insufficiency and hence started on Inj. Hydrocortisone $100 \mathrm{mg}$ iv Q6H. Patient's BP improved to normal levels.

As part of workup for adrenal insufficiency, hormone studies were done. Thyroid hormone, FSH, LH, Prolactin and Estradiol levels were found to be low. Hence, hypopituitarism was considered and MRI Brain was done, which revealed partial empty sella with small pituitary gland. Hence, with the clinical history and investigatory findings, patient was diagnosed as having secondary adrenal insufficiency due to pituitary apoplexy (possible Sheehan's syndrome). Patient was instituted on oral glucorticoids, thyroxine and estrogen supplements. She clinically improved, laboratory parameters showed increasing trend and was hence discharged.

\section{Discussion}

Adrenal insufficiency is a disorder caused due to the deficiency of predominantly glucocorticoid and sometimes mineralocorticoid deficiency. It may present with a wide array of symptoms and signs. It can be classified into primary, secondary or tertiary based on the etiopathogenesis of the disease. It is known as primary, if the condition arises due to pathology of the adrenal gland, secondary, if there is interference with ACTH secretion by the pituitary gland, and tertiary, due to interference in the corticotropin releasing hormone ( $\mathrm{CRH})$ by the hypothalamus. Adrenal crisis may present with hypotension refractory to fluids, hyponatremia, vomiting, fever and mental status changes. This is usually precipitated by stress, infection or surgery, and in patients who are abruptly withdrawn from glucorticoid therapy. The main symptoms and signs of chronic adrenal insufficiency include fatigue, lassitude, anorexia, nausea, vomiting, weight loss, giddiness, orthostatic hypotension, hyperpigmentation, dyselectrolytemia, sexual dysfunction, auricularcartilage calcification and neuropsychiatric manifestations.

Despite their physiologic differences, the psychiatric presentation of Addison's disease may be similar to that of Cushing's syndrome. Presenting symptoms include delusions, hallucinations, depression, apathy, social withdrawal, poverty of thought, and negativism. A true organic psychosis can also occur, characterized by cognitive impairment and confusion, and in certain cases stupor or coma. The relationship of these symptoms to decreased 
levels of glucocorticoids is clear in that there is a quick resolution with glucocorticoid replacement. Correction of the electrolyte imbalance does not by itself result in observable benefit. If psychiatric symptomatology is profound and antedates the more classic somatic features, misdiagnosis may occur. In our case, patient was misdiagnosed as having psychiatric disorder after having presented with a history of fatigue, impaired sleep, delusions and irritable behavior. Anglin et al noted four case series published in the 1940s and 1950s that found the prevalence of neuropsychiatric symptoms in adrenal insufficiency to be between 64 and 84 percent. Iwata et al reported that in some cases, the neuropsychiatric symptoms were the initial and sole presentation, even though such symptoms are more common in the late course of the disease; this might lead to a patient initially being misdiagnosed, as it did in our case, and in turn, incorrectly treated.

Concerning the etiology of neuropsychiatric symptoms in $\mathrm{AD}$, many theories exist. Obviously, the first causes that come to mind would be the electrolytic and metabolic disturbances that are commonly associated with AD. Hyponatremia being the cardinal sign of Adrenal crisis could play a role in brain damage. In a study by Engel and Margolin, attention was directed to the electrical activity of the brain, where five out of eight AD patients had abnormal electroencephalograms (EEGs). In the same study, the correlation between hypoglycemia and EEGs was observed. To be more specific, in the case report by Anglin et al, the patient's initial EEG during an Adrenal crisis showed fluctuating slow activity, with 1- to 3- second bursts of slow activity, although this improved later during the patient's follow-up period. Another hypothesis concerning the etiology of neuropsychiatric symptoms would be the effect of glucocorticoids on the brain and cognitive function. Henkin proposed that a decrease in glucocorticoids results in an increase in neural excitability, leading to an enhanced ability to detect sensory input. It has been postulated that a decrease in glucocorticoids could precipitate hallucinations as well as lower the threshold for psychosis. In a more recent study by Yehuda and Seckl, the authors proposed that low cortisol levels may affect vulnerability to psychosis. Ironically, prolonged corticosteroid therapy, the mainstay therapy for adrenal insufficiency patients, can cause neuropsychiatric symptoms. Some of the side effects like mood alterations, hyperactivity, insomnia, suicidal tendencies and psychosis have been described in approximately five percent of corticosteroidtreated patients. The theory that a past history of psychiatric illness is a risk factor for corticosteroid-induced psychosis is controversial, as studies claim that it does not appear to impose a significant risk factor.

\section{Conclusion}

It is important that physicians are aware of the neuropsychiatric symptoms that can manifest in Adrenal insufficciency patients because sometimes these symptoms are the earliest manifestations of a life-threatening Adrenal crisis. This should always be kept in mind while treating a psychiatric patient in the out patient setting, especially in cases of depression and psychosis, without a prior family history of psychiatric illness or those showing poor response to pharmacological therapy. We present this case to highlight the atypical clinical manifestation of adrenal insufficiency, which had masqueraded as psychiatric illness and deluded apt pharmacological intervention.

\section{References}

1. Anglin RE, Rosebush PI, Mazurek MF The neuropsychiatric profile of Addison's disease: revisiting a forgotten phenomenon. Journal of neuropsychiatry and clinical neurosciences [2006, 18(4): 450-459]

2. CM Brosnan, NFC Gowing, Lesson of the Week: Addison's disease, British Medical Journal 1996 - bmj.com 312.7038.1085 
3. Smith, Kent C. M.D.; Barish, Joel M.D.; Correa, Javier M.D.; Williams, Robert H. M.D. , Psychiatric Disturbance in Endocrinologic Disease, Journal of psychosomatic medicine, January 1972 Volume 34 - Issue 1

4. E. Ur, T. H. Turner, T. J. Goodwin, A. Grossman, G. M. Besser, Mania in association with hydrocortisone replacement for Addison's disease, Postgrad Med J 1992;68:41-43

5. Behavioral Disturbances Associated with Endocrine Disorders, Annual Review of Medicine, Vol. 37: 205-214 (Volume publication date February 1986)

6. R Baschetti , Chronic fatigue syndrome: a form of Addison's disease - Journal of internal medicine, 2000 - Wiley Online Library

7. Varadaraj, R.; Cooper, A. J., Addison's disease presenting with psychiatric symptoms, The American Journal of Psychiatry, Vol 143(4), Apr 1986, 553554.

8. Anders F. Thomsen, Tine K. Kvist, Per K. Andersen, Lars V. Kessing, The risk of affective disorders in patients with adrenocortical insufficiency, Psychoneuroendocrinology Volume 31, Issue 5, June 2006, Pages 614-622 